\title{
Acute and convalescent changes in plasma homocysteine concentrations in acute coronary syndromes
}

\author{
M K Al-Obaidi, P J Stubbs, R Amersey, M I M Noble
}

\begin{abstract}
Background-Raised plasma homocysteine is a risk factor for coronary artery disease. Patients with myocardial infarction or unstable angina show greater activation of coagulation, greater troponin release, and a worse outcome.

Objective-To examine variations in plasma homocysteine concentration in relation to $\mathrm{C}$ reactive protein (CRP) in patients presenting with acute coronary syndromes.

Methods-Consecutive patients presenting with acute myocardial infarction (22) and unstable angina pectoris (12) were studied. Plasma samples were obtained on admission (before clinical intervention), on days 2,7 , and 28, and again six months after admission. Plasma homocysteine, assayed by high performance liquid chromatography, and CRP were both determined at the same time points. Changes were assessed by analysis of variance.

Results-CRP concentrations showed a classical rise on day 2 , followed by a gradual decline to normal values taken at six months from admission in both myocardial infarction $(\mathrm{p}<0.0001)$ and unstable angina $(\mathrm{p}=0.02)$. Homocysteine concentrations in myocardial infarction (median, 25th to 75 th interquartile range) were: 11.9 (10.7 to 12.6$), 11.5$ (9.1 to 13.4$), 12.1$ (11.4 to 14.1 ), 12.4 (11.1 to 14.4 ), and 12.1 (11.2 to 14.0$) \mu \mathrm{mol} / 1$, for days $1,2,7,28$, and 180 , respectively $(p=0.02)$. Significant differences were observed only between day 2 and day $7(p<0.05)$. The final homocysteine measurement was not different from the admission level. Homocysteine concentrations in unstable angina did not differ between admission and convalescence (12.5 (9.1 to 14.5$) \mu \mathrm{mol} / 1$ and 12.3 (7.7 to 14.9$) \mu \mathrm{mol} / 1$, respectively).

Conclusions-Plasma homocysteine concentrations are minimally influenced by acute phase variations with reliable measurements obtained on admission in patients with myocardial infarction and unstable angina.

(Heart 2001;85:380-384)
\end{abstract}

Keywords: myocardial infarction; unstable angina; homocysteine; sample timing

Risk stratification following acute coronary events is integral to current management protocols for acute coronary syndromes of myocardial infarction and unstable angina pectoris. However, biochemical assays performed during the acute event have been known to give false high or low results as a result of the acute phase reactions. ${ }^{1}$ As an example, reliable estimates of total cholesterol can be obtained within 12-24 hours of the onset of symptoms, but concentrations fall by $20-40 \%$ on days $4-5$, and subsequent estimates may not be reliable again until two months after the event. ${ }^{2}$

It has recently been shown that a moderate increase in plasma homocysteine is independently associated with the development of atherothrombotic vascular disease..$^{3-5}$ While trials are currently under way to evaluate the benefit of homocysteine lowering treatment on risk modification, this potential benefit has not been considered in patients presenting with acute vascular events. We have recently shown that raised plasma homocysteine concentrations influence thrombosis during acute coronary events. ${ }^{6}$ Furthermore, patients with increased homocysteine are at significantly greater risk of long term mortality. ${ }^{7}$ These results suggest that homocysteine lowering treatment may need to be considered during risk stratification after acute coronary syndromes.
In healthy individuals, plasma homocysteine concentrations measured over one month and 30 months show high reliability coefficients of 0.94 and 0.65 , respectively. ${ }^{8}$ Variations in plasma homocysteine concentrations following acute vascular events have only been sparsely reported. In these studies, however, sampling from the patients was undertaken at least 24-48 hours after the onset of the clinical event.

To evaluate whether admission levels offer a reliable measure of homocysteine concentration, we present data on the variations in plasma homocysteine concentrations in patients presenting with acute coronary syndromes in whom baseline sampling was achieved on admission and before clinical intervention.

\section{Methods}

STUDY DESIGN AND SAMPLE SIZE

In view of the difficulty in obtaining blood samples before thrombolysis in patients admitted to hospital with acute coronary syndromes, we decided on a paired design. A paired design restricts the measurements to multiple estimations over time in the same patient, so that the factor that contributes to the statistical significance is whether the variable goes up or down, and not whether variances at different 
times overlap (unpaired design). For small changes, an unpaired design requires a very large sample size. With a paired design, only 10 subjects would have been required in this study. However, we saw no reason to exclude any of the 12 patients with unstable angina or the 22 with myocardial infarction whom we were able to recruit.

\section{STUDY POPULATION}

Patients with acute coronary syndromes were consecutively recruited on admission to a single coronary care unit in a London teaching hospital. Informed consent was obtained from all the patients, with the approval of the local ethics committee. The patients were given a provisional diagnosis to guide appropriate treatment, but this diagnosis was subsequently reviewed during follow up, and the final diagnosis (myocardial infarction and unstable angina) was used in all analyses.

Myocardial infarction was diagnosed according to the World Health Organization criteria. ${ }^{9}$ Unstable angina was diagnosed if the WHO criteria for myocardial infarction were not met and if all enzyme measurements (creatine kinase, aspartate transaminase, hydroxybutyrate dehydrogenase) were below twice their upper reference range throughout the routine sampling period (daily for three days). Evidence of ischaemic heart disease was demonstrated by either a follow up cardiac event (death, coronary revascularisation, or myocardial infarction), a positive exercise treadmill test $(>0.1 \mathrm{mV}$ ST segment depression $80 \mathrm{~ms}$ after the J point), or the demonstration of ischaemia on thallium radioisotopic scanning.

CLINICAL DATA

Full details of the clinical features, investigations, and management were recorded on all patients. Clinical details included risk factor assessment for coronary artery disease. Smoking status was classified as current smokers and non-smokers; current smokers included individuals who had stopped smoking less than four weeks before enrolment into the study. A known history of hypertension or diabetes mellitus, and details of treatment received before admission, was also recorded. Routine lipid profile estimation was carried out on admission in all the patients, documenting their total cholesterol, triglycerides, and high density lipoprotein (HDL) concentrations.

Table 1 Clinical and risk profile characteristics of the study groups

\begin{tabular}{lcc}
\hline & $\begin{array}{c}\text { Myocardial infarction } \\
(n=22)\end{array}$ & $\begin{array}{l}\text { Unstable angina } \\
(n=12)\end{array}$ \\
\hline Age (years) (mean (SEM)) & $63(2)$ & $61(3)$ \\
Sex (male:female) & $13: 9$ & $4: 8$ \\
Diabetes mellitus & 2 & 2 \\
Total cholesterol > 6 mmol/1 (n) & 13 & 6 \\
HDL > 1.0 mmol/1 (n) & 9 & 6 \\
Treated with statins on discharge (n) & 16 & 7 \\
Smoking (n) & 13 & 11 \\
$\quad$ Current & 9 & 1 \\
$\quad$ Non-smoker & $98.4(4.2)$ & $106.3(6.0)$ \\
Creatinine $(\mu \mathrm{mol} / \mathrm{l})$ (mean (SEM)) & &
\end{tabular}

HDL, high density lipoprotein.
PROTOCOL

Venous blood samples were obtained on admission by trained medical or senior nursing staff from a cuffed antecubital vein before initiation of thrombolysis or anticoagulant treatment. Blood was transferred into bottles containing EDTA (7.5\%; Becton Dickinson, Meylan cedex, France) for homocysteine assay, and into bottles containing no anticoagulant for $\mathrm{C}$ reactive protein (CRP) assay. Within 15 minutes of collection, platelet-poor plasma/ serum was then obtained by centrifugation at room temperature for 15 minutes at $3000 \mathrm{~g}$, and immediately transferred to a $-80^{\circ} \mathrm{C}$ freezer.

Serial samples were then obtained from each patient on day 2 , day 7 , and day 28 , and six months (180 days) after the acute event. The latter three time points were arranged, when possible, to coincide with routine follow up assessments. Samples were collected between 09.00 and 10.00 hours in the fasting state, and plasma/serum samples were obtained and stored as described above. Blind analysis of all samples was performed in batches on the completion of sample collection.

HOMOCYSTEINE MEASUREMENTS

Plasma total homocysteine, which includes the sum of protein bound and free homocysteine, was measured by high performance liquid chromatography with fluorescence detection. ${ }^{11}{ }^{11}$ The intra- and interassay coefficients of variation were $5 \%$ or less. Plasma homocysteine was recorded in units of $\mu \mathrm{mol} / 1$.

CRP MEASUREMENTS

CRP assay was performed with the Behring Nephelometer II using the Behring latex enforced immunophelometric kit (Behring UK, Milton Keynes, Buckinghamshire, UK), according to the manufacturer's recommended protocol. The interassay coefficient of variation was less than $3 \%$.

STATISTICAL METHODS

Values are expressed as median (25th to 75 th interquartile range). Serial homocysteine values were compared by non-parametric analysis of variance (ANOVA) for repeated measures (Freidman) and Dunn's test for post hoc analysis. Variations in plasma homocysteine are expressed as percentage change from the admission value. Correlations were tested by Spearman rank test and correlation coefficient $\left(r_{\mathrm{s}}\right)$. Reliability coefficient $(R)$ was tested as follows: $R=\left(\mathrm{S}^{2} \mathrm{BP} / \mathrm{S}^{2}\right.$ Total $)=$ $\mathrm{S}^{2} \mathrm{BP} /\left(\mathrm{S}^{2} \mathrm{BP}+\mathrm{S}^{2} \mathrm{WP}\right)$, where $\mathrm{S}^{2} \mathrm{BP}$ is the between person variance, $S^{2} \mathrm{WP}$ is the within person variance, and $\mathrm{S}^{2}$ Total is the total variance.

\section{Results}

Curves were available on 34 patients, 22 with myocardial infarction and 12 with unstable angina. Table 1 shows the clinical characteristics of the study groups. Patients with unstable angina had a higher incidence of previous ischaemic heart disease but otherwise had a similar risk factor profile to patients with 
Table 2 Follow up events and clinical interventions in the study group

\begin{tabular}{lll}
\hline & $\begin{array}{l}\text { Myocardial infarction } \\
(n=22)\end{array}$ & $\begin{array}{l}\text { Unstable angina } \\
(n=12)\end{array}$ \\
\hline Thrombolysis & 13 & - \\
Q wave infarction & 10 & - \\
Anticoagulation & 22 & 12 \\
EST & 5 & 4 \\
$\quad$ Positive & 13 & 7 \\
$\quad$ Negative & 4 & 1 \\
Inconclusive & 9 & 7 \\
Angiography & 1 & 5 \\
PTCA & 1 & 0 \\
CABG list & &
\end{tabular}

Values are numbers of patients.

CABG, coronary artery bypass graft surgery; EST, electrocardiographic exercise stress test (usually carried out 3-4 weeks after the acute coronary event); PTCA, percutaneous transluminal coronary angiography.

myocardial infarction. Table 2 shows the interventions, investigations, and management decisions during the study period. At the six months follow up, all patients had been stabilised either with medical treatment or with invasive intervention (percutaneous transluminal coronary angioplasty). One patient was put on the waiting list for coronary artery bypass graft surgery but was asymptomatic on medical treatment at six months. No patient was receiving treatment with supplementary vitamins during the study period.

After the onset of chest pain (defined as the first or most severe episode of chest pain before admission), patients with unstable angina presented later than those with myocardial infarction: mean (SD) 14.3 (7.1) hours, range
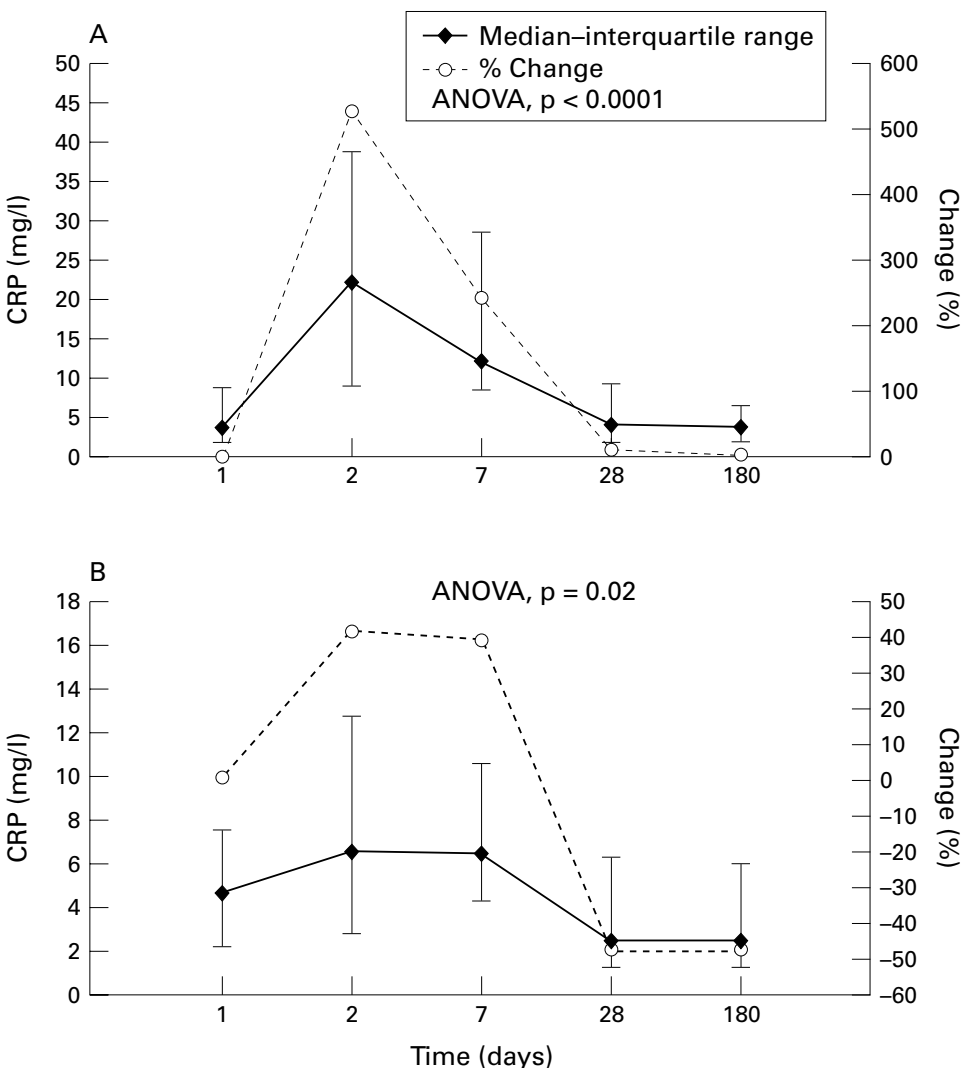

Figure $1 C$ reactive protein $(C R P)$ variations following $(A)$ myocardial infarction $(p<0.0001)$, and $(B)$ unstable angina pectoris $(p=0.02)$. Continuous lines represent the median with 25 th to 75 th interquartile ranges; interrupted lines represent the percentage change.
0.45-72 hours, v 3.7 (1.2) hours, range $0.30-15$ hours; $p<0.05$.

Figure 1A shows the variations in CRP concentrations following myocardial infarction. There was a classical rise, reaching a peak on day 2 and declining to normal values by day 180 ( $\mathrm{p}<0.0001$, ANOVA). A similar pattern was observed in patients with unstable angina; however, peak CRP concentrations (on day 2) were significantly lower in the unstable angina group than in the patients with myocardial infarction, at 6.4 (3.9 to 11.3$) \mathrm{mg} / \mathrm{l} v 22.2$ (8.9 to 39.7) $\mathrm{mg} / \mathrm{l}$, respectively $(\mathrm{p}=0.005)$. There were no differences between admission and final CRP values in either myocardial infarction or unstable angina.

Figure 2A shows the variations in plasma homocysteine concentration following myocardial infarction. In comparison with the admission concentration, there was a minor fall $(4 \%)$ on day 2 , followed by a rise on day 7 by $2 \%$. The final homocysteine measurement on day 180 was $2 \%$ higher than on admission $(p=0.02$, ANOVA). Dunn's post hoc analysis showed significant differences only between day $2(11.5$ (9.1 to 13.4$) \mu \mathrm{mol} / \mathrm{l})$ and day 7 (12.1 (11.4 to 14.1$) \mu \mathrm{mol} / \mathrm{l})(\mathrm{p}=0.05)$. Correlation analysis revealed a significant correlation between homocysteine concentrations on admission and on day $2(r=0.66, \mathrm{p}<0.001)$, day $7(r=0.70, \mathrm{p}<0.001)$, day $28(r=0.70$, $\mathrm{p}<0.001)$, and day $180(r=0.57, \mathrm{p}=0.005)$. The homocysteine measurements were consistently reliable throughout the study period ( $R=0.89,0.89,0.89,0.87$, and 0.83 for days $1,2,7,28$, and 180 , respectively).

Patients with unstable angina (fig 2B) had a $2 \%$ fall in homocysteine concentrations on day 2 , and the final homocysteine measurement on day 180 was only $2 \%$ lower than on admission. There were significant correlations between the values on day 1 and day $2 \quad(r=0.85$, $\mathrm{p}<0.001)$, between day 1 and day $7(r=0.80$, $\mathrm{p}=0.002)$, between day 1 and day 28 $(r=0.83, \mathrm{p}<0.001)$, and between day 1 and day $180(r=0.78, \mathrm{p}=0.003)$. High reliability coefficients for those time points were also observed $(R=0.83,0.83,0.89,0.83$, and 0.85 , respectively).

\section{Discussion}

This is the first report of measurements of homocysteine on admission in patients with acute coronary syndromes. It is also larger than the previous study of serial measurements, ${ }^{12}$ in which the observations began on day 2 . That study therefore missed the fall in plasma homocysteine concentration from day 1 to day 2 . The subsequent rise from day 2 onwards was similar in both studies.

CRP variations occur very early after the onset of chest pain in patients with acute myocardial infarction, concentrations almost doubling within the first eight hours. Peak concentrations are usually reached by days $2-3$ and decline rapidly after that. ${ }^{13}$ In our study, CRP values on admission were slightly higher (though the difference was not significant) in patients with unstable angina than in those with myocardial infarction. This may reflect 

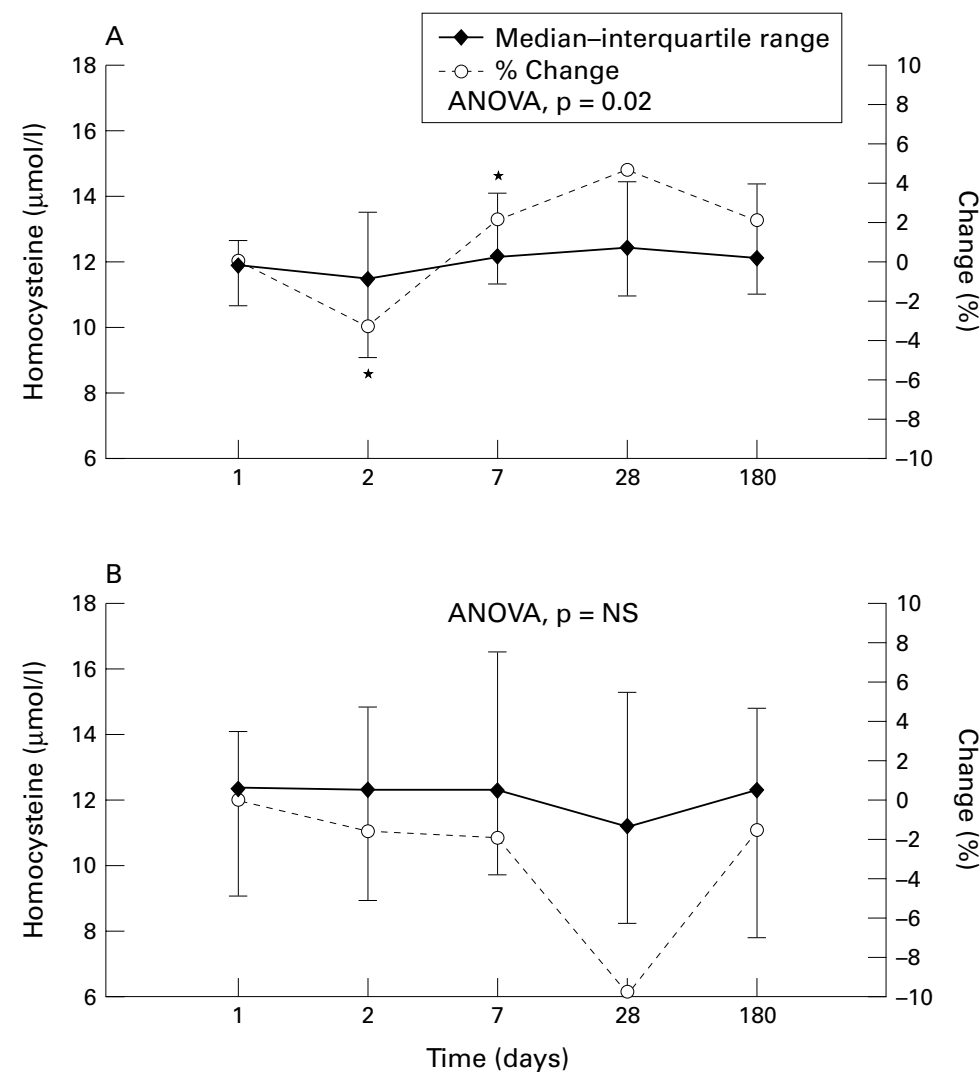

Figure 2 Plasma homocysteine variations following (A) myocardial infarction $(p=0.02)$, and $(B)$ unstable angina pectoris (NS). Continuous lines represent the median with 25 th to 75 th interquartile ranges; interrupted lines represent the percentage change. ${ }^{\star}$ Dunn's post hoc analysis, $p<0.05$.

the later presentation of patients with unstable angina following the onset of chest pain. There were no differences in CRP concentrations between the admission values and the final measurement taken six months later in both groups. This is important for two reasons: first, it is necessary to establish that the initial sample was taken at a time when the acute phase reaction was at its early stages; and second, the convalescent sample needs to be taken when the acute phase reaction has settled to normal values. Homocysteine concentrations determined during convalescence could therefore be taken to represent a reliable estimate for each individual patient.

Homocysteine concentrations have been reported to be higher by $4 \%$ at six weeks, ${ }^{14}$ by $19 \%$ at six months, ${ }^{12}$ and by $27 \%$ at 1.5 years, ${ }^{15}$ compared with initial values determined between 24-48 hours after onset of an acute vascular event. These results contrast with our observations of only a $2-3 \%$ difference in homocysteine concentration between convalescent values and admission concentrations. The maximum variability in homocysteine concentrations was $7 \%$ and occurred between day 2 (peak fall) and day 28 (peak rise) after the onset of chest pain. This represented a much lower variation than observed with plasma lipids $(40-60 \%)$ in similar patient groups. ${ }^{16-18}$

Our study is the first to describe plasma homocysteine variations with sampling achieved at the earliest possible time point of clinical presentation. The nature of the patients studied and the characteristics of the clinical settings, however, limit the standardisation of patients in terms of their fasting/non-fasting status. It is well known that homocysteine concentrations rise following a methionine load, for example after a high protein meal. A confounding factor lies in the clinical setting of acute coronary ischaemia, where the majority of patients experience some degree of nausea with or without vomiting; this may in turn lead to a negative methionine balance and enhancement of remethylation of homocysteine to methionine. ${ }^{19}$ Despite these considerations, our results show that in patients with acute coronary syndromes there was no difference between plasma homocysteine concentrations on admission and after a long period of convalescence, when the estimations were made in a standardised fasting state and with no evidence of a systemic inflammatory reaction. Furthermore, even when considering the first fasting sample (the second time point in our study), homocysteine concentrations were not significantly different from convalescent values.

These data should enable the risk associated with the prothrombotic properties of homocysteine to be reliably assessed. In a previous study, ${ }^{6}$ we found that high admission plasma homocysteine concentrations were associated with increased thrombin formation, as judged by prothrombin fragments $F 1+2$. Evidence that hyperhomocysteinaemia was triggering the thrombosis cascade was obtained from raised concentrations of activated factor VII (factor VIIa), the product of the reaction of factor VII with tissue factor. Coronary angiography was only carried out in about half the patients, but there were no differences between groups in the number of major coronary arteries with disease.

The main outcome of our study is that admission homocysteine concentrations are reliable for risk stratification purposes, and that homocysteine estimation within the first 28 days from the onset is only slightly influenced by acute phase reactions.

This study was supported by a concerted action grant of the Biomed 1 Program of the European Commission, No PL92005.

1 Vetter NJ, Strange RC, Adams W, et al. Initial metabolic and hormonal response to acute myocardial infarction. Lancet $1974 ; \mathrm{i}: 284-8$

2 Gore JM, Goldberg RJ, Matsumoto AS, et al. Validity of serum total cholesterol level obtained within 24 hours of
acute myocardial infarction. Am f Cardiol 1984;54:722-5. 3 Clarke R, Daly L, Robinson K, et al. Hyperhomocysteinemia: an independent risk factor for Hyperhomocysteinemia: an independent risk factor

4 Stampfer MJ, Malinow MR, Willett WC, et al. A prospective Stampfer MJ, Malinow MR, Willett WC, et al. A prospective
study of plasma homocyst(e)ine and risk of myocardial infstudy of plasma homocyst(e)ine and risk of myocard
arction in US physicians. $\mathscr{F} A M A 1992 ; 268: 877-81$.

5 Perry IJ, Refsum H, Morris RW, et al. Prospective study of serum total homocysteine concentration and risk of stroke in middle-aged British men. Lancet 1995;346:1395-8.

6 Al-Obaidi MK, Phillippou H, Stubbs PJ, et al. Relationships between homocysteine, factor VIIa and thrombin generation in acute coronary syndromes. Circulation 2000;101: 372-7.

7 Stubbs PJ, Al-Obaidi MK, Conroy R, et al. The effect of plasma homocysteine concentration on early and late events in patients with acute coronary syndromes. Circulation 2000;102:605-10.

8 Garg UC, Zheng ZJ, Folsom AR, et al. Short-term and long-term variability of plasma homocysteine measurelong-term variability of plasma
ment. Clin Chem 1997;43:141-5.

9 WHO. Working group on the establishment of ischaemic heart disease registers: Report of the fifth working group. Geneva: WHO, 1971. 
10 Fiskerstrand T, Refsum H, Kvalheim G, et al. Homocysteine and other thiols in plasma and urine: automated determi-

11 Refsum H, Ueland PM, Svardal AM. Fully automated fluorescence assay for determining total homocysteine in plasma. Clin Chem 1989;35:1921-7.

12 Egerton W, Silberberg J, Crooks R, et al. Serial measures of plasma homocyst(e)ine after acute myocardial infarction. Am $\mathcal{F}$ Cardiol 1996;77:759-61.

13 de Beer FC, Hind CR, Fox KM, et al. Measurement of serum C-reactive protein concentration in myocardial ischaemia and infarction. Br Heart f 1982;47:239-43.

14 Landgren F, Israelsson B, Lindgren A, et al. Plasma homocysteine in acute myocardial infarction: homocysteinelowering effect of folic acid. F Intern Med 1995;237:381-8.
15 Lindgren A, Brattstrom L, Norrving B, et al. Plasma homocysteine in the acute and convalescent phases after stroke.

16 Fyfe T, Baxter RH, Cochran KM, et al. Plasma-lipid changes after myocardial infarction. Lancet 1971;ii:9971001 .

17 Avogaro P, Bon GB, Cazzolato G, et al. Variations in apolipoproteins $\mathrm{B}$ and $\mathrm{A} 1$ during the course of myocardial infarction. Eur $\mathcal{F}$ Clin Invest 1978;8:121-9.

18 Heldenberg D, Rubinstein A, Levtov O, et al. Serum lipids and lipoprotein concentrations during the acute phase of myocardial infarction. Atherosclerosis 1980;35:433-7.

19 Hiramatsu T, Fukagawa NK, Marchini JS, et al. Methionine and cysteine kinetics at different intakes of cystine in healthy adult men. Am f Clin Nutr 1994;60:525-33.

\section{IMAGES IN CARDIOLOGY}

\section{A subtle sign of aortic outflow obstruction in an infected 29 year old Starr-Edward's valve}

A 70 year old man had a Starr-Edward's aortic valve prosthesis inserted in 1970 for aortic stenosis. He presented 29 years later with enterococcus septicaemia, acute renal failure, and cardiac failure requiring ionotropes.

Transoesophageal echocardiography (TOE) showed no evidence of aortic outflow obstruction, valvar incompetence, or a paravalvar leak to explain the gross heart failure. A left heart catheter showed normal coronary arteries. Closer inspection of the movement of the ball within the cage, however, showed it to be skewed and asymmetrical (below left). We suspected the presence of infected debris within the cage and therefore proceeded to surgery on the basis that there was outflow obstruction.

At surgery there was no debris within the cage itself. However half of the undersurface of the valve orifice was occluded (below centre).
Blood was flowing through the patent half and pushing the ball to the other side of the cage (below right). There was therefore a significant outflow obstruction, which explained the patient's cardiac failure and the abnormal movement of the ball at angiography.

The abscess was debrided, the annulus reconstructed with bovine pericardium, and an Edwards Lifesciences perimount valve was inserted. The patient's heart failure resolved postoperatively. He was discharged on day 35 .

This case illustrates the limitations of TOE in the management of prosthetic valve endocarditis. It also demonstrates a subtle sign of obstruction in a Starr-Edward's aortic prosthetic valve.

E F AKOWUAH

C V P ONYEAKA

G J COOPER

mail@grahamcooper.freeserve.co.uk
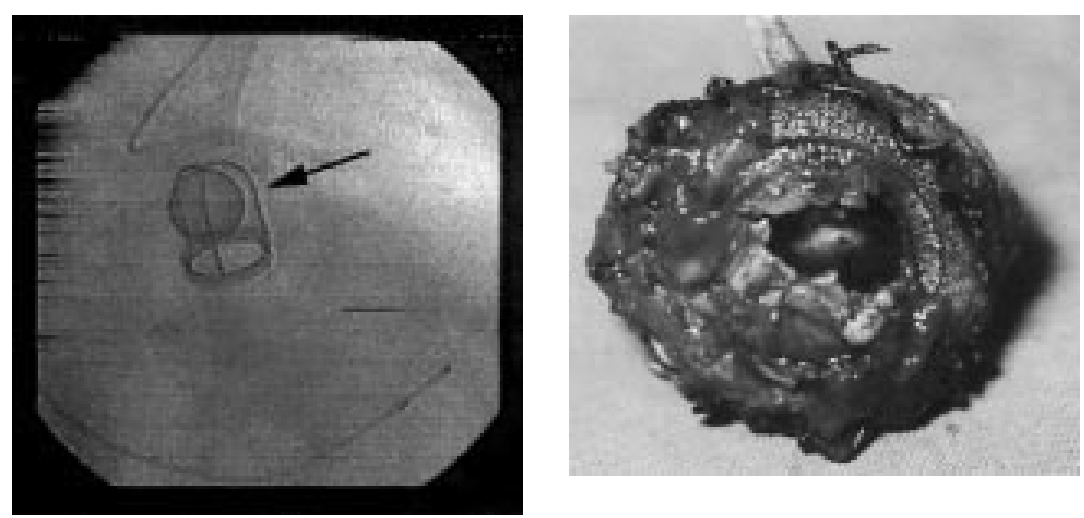

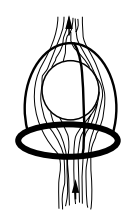

Flow through a normal Starr-Edward's prosthetic valve
B

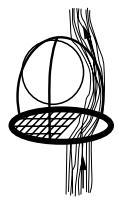

Flow through the Starr-Edward's prosthetic valve with a partially occluded valve ring. Blood flows through the open side at the base, pushing the ball to the opposite side. 